\title{
Making compact elliptical satellite galaxies: a conceptual model for M32
}

\author{
John S. Gallagher, III ${ }^{1}$, Eva K. Grebel ${ }^{2}$ and Linda J. Smith \\ ${ }^{1}$ Dept. Astronomy, U. Wisconsin, 475 N. Charter St. Madison, WI 53760, USA \\ email: jsg@astro.wisc.edu \\ ${ }^{2}$ Astronomisches Institut, Venusstrasse 7, CH-4102, Binningen, Switzerland \\ ${ }^{3}$ Dept. Physics \& Astronomy, Gower St., U. College London, London WC1E 6BT, UK
}

\begin{abstract}
M32 and other compact dwarf galaxies present interesting evolutionary puzzles. Why are most dwarf galaxies low density systems while a very few, like M32, have the highest stellar densities of any galaxies? We suggest that gas capture should be added to the tidal stirring mix as a way to make high density M32-type dwarfs starting with relatively ordinary satellite galaxies. This paper presents some of the basic features and issue associated with gas capture models.
\end{abstract}

Keywords. galaxies: dwarf, galaxies: evolution, galaxies: individual (M32, NGC 1510), galaxies: structure

\section{Introduction}

The formation of galaxies requires the production of appropriately massive gravitationally bound structures and gas dissipation to make the stars which inhabit all galaxies. In this regard lower mass galaxies present an interesting diversity, as they commonly include the least dense stellar systems, which are inferred to have experienced minimal dissipation. Thus the dSphs may sit on the boundary between objects which become galaxies, and those which fail. Even though the dEs, such as the NGC 147-185-205 satellites of M31 have higher surface densities than the dSphs, they are still relatively puny systems. The rare compact dwarf ellipticals, such as M32, however, sit at the other extreme and are in effect high density galactic cannon balls. This paper briefly presents some ideas about how such high density galaxies might evolve and discusses why they are important.

M32 and the dE NGC205 have similar optical luminosities $\left(\mathrm{M}_{V} \approx-16\right)$ and thus contain roughly the same stellar masses $\left(\sim 10^{9} \mathrm{M}_{\odot}\right)$. M32 is much more compact with an effective radius that is 0.18 that of NGC 205. The central density of M32 exceeds that of the NGC 205 star cluster nucleus by a factor of $\sim 100$ (Choi et al. 2002) and it even lies above the central densities of M31 and M33 (Lauer et al. 1998). M32 likely falls near the maximum stellar density limit for galaxies, and probably has a $\sim 10^{6} \mathrm{M}_{\odot}$ nuclear black hole (Joseph et al. 2001).

Despite its unusual structure, M32 seems to have had a comparatively normal star formation history. Star formation in its central region probably continued until 3-5 Gyr ago, while the outer stellar populations tend to be older (Rose et al. 2005, Worthey 2004, Worthey et al. 2004). A range in mean stellar metallicity exists, falling from near solar at the nucleus to modestly subsolar at larger radii. The abundance ratio of the $\alpha$-element $\mathrm{Mg}$ to $\mathrm{Fe}$ is solar or below. The extended period of star formation in M32 is typical of dEs, with the caveat that M32 has higher stellar metal abundances relative to its current stellar mass. 
How M32, a galactic "swan," ended up among the ducks and geese of the M31 companion dSphs and dEs is a long standing puzzle. One constraint is set by the rarity of M32-type galaxies; their production should be uncommon and/or they should be shortlived. The existing models can be roughly divided into three general classes:

- Formation oddities: M32 owes its existence to highly unusual conditions at the time of its formation. For example, Burkert (1994) suggested that it could be the result of the collapse of a galaxy within the pre-existing tidal field of a giant galaxy.

- Transformation of a normal galaxy: This has historically been the most popular model for M32 (Faber 1973; see also Nieto \& Prugniel 1987, Choi et al. 2002 and references therein). Recent extensions of this model include the harassed spiral picture developed by Bekki et al. (2001).

- Mergers of star clusters: If dense star clusters formed in a starburst clump merge, then they can make some classes of ultra-compact dwarfs (e.g., Fellhauer \& Kroupa 2005). However, M32 seems too massive for this path to apply; one would need to merge thousands of super star clusters to make M32.

Here we start from the fundamental concepts of the Nieto \& Prugniel (1987) model and also consider the role of gas capture in the evolution of dwarf companion galaxies. At the outset we should emphasize that more than one path may exist to the production of compact E dwarfs; one model may not fit all cases (see $\S 4$ and Bekki et al. 2001 for discussion of issues).

\section{Gas Accretion and Starbursts in Dwarf Satellites}

While gas loss from dwarfs orbiting in the halos of giant galaxies are frequently discussed, the possibility that some dwarf companions can capture gas from their giant hosts receives less attention. Hawarden et al. (1979) invoked gas capture by NGC 1510, a satellite interacting with the spiral NGC 1512, to explain the disturbed nature of NGC 1510. Their model assumed Bondi-Hoyle accretion, $d M_{g} / d t \propto M_{\text {sat }}^{2} \overline{\rho_{I S M}} / V_{\text {rel }}^{3}$ where $\overline{\rho_{I S M}}$ is the mean galactic ISM gas density and $V_{\text {rel }}$ is the velocity of the satellite through the ISM. Thus high gas densities and, especially, low $V_{\text {rel }}$ favor accretion. However, galaxies moving with $V_{\text {rel }}$ that are characteristic of galactic orbital velocities probably will be stripped rather than accrete gas (e.g., the Magellanic Stream).

The optical structure of the NGC 1510/12 system shows that the gas-accretion situation may be more favorable than suggested by the Bondi-Hoyle models. A tidal arm appears to extend from NGC 1512 to NGC 1510 (Figure 1), a circumstance that is seen in other interacting starburst satellites, including NGC 3077, the starburst companion to M81. Presumably these interacting satellites are on eccentric prograde orbits with low inclinations where coupling to the disk of the giant system is enhanced (e.g., Walker et al. 1996). The observations further suggest that matter from the arm is ending up in the dwarf. This process resembles the assembly of a new dwarf galaxy in a tidal tail as described by Duc et al. (2004), with the added advantage that a gravitationally bound object is already present to collect any gas that flows into the area.

A process that fosters intense modes of star formation also is needed if we are to achieve high stellar densities. Fortunately the observations suggest that star formation in accreting dwarfs is usually intense and centrally concentrated (e.g., Figure 2). This effect may be aided by the tendency for interacting disk galaxies to form transient bars (Miwa \& Noguchi 1997), which funnel gas inwards and feed nuclear starbursts. Furthermore, in orbiting galaxies we expect multiple starbursts associated with peri-galacticon passages (Kravtsov et al. 2004). The combination of the possible loss of ejecta from SNe II (e.g., 


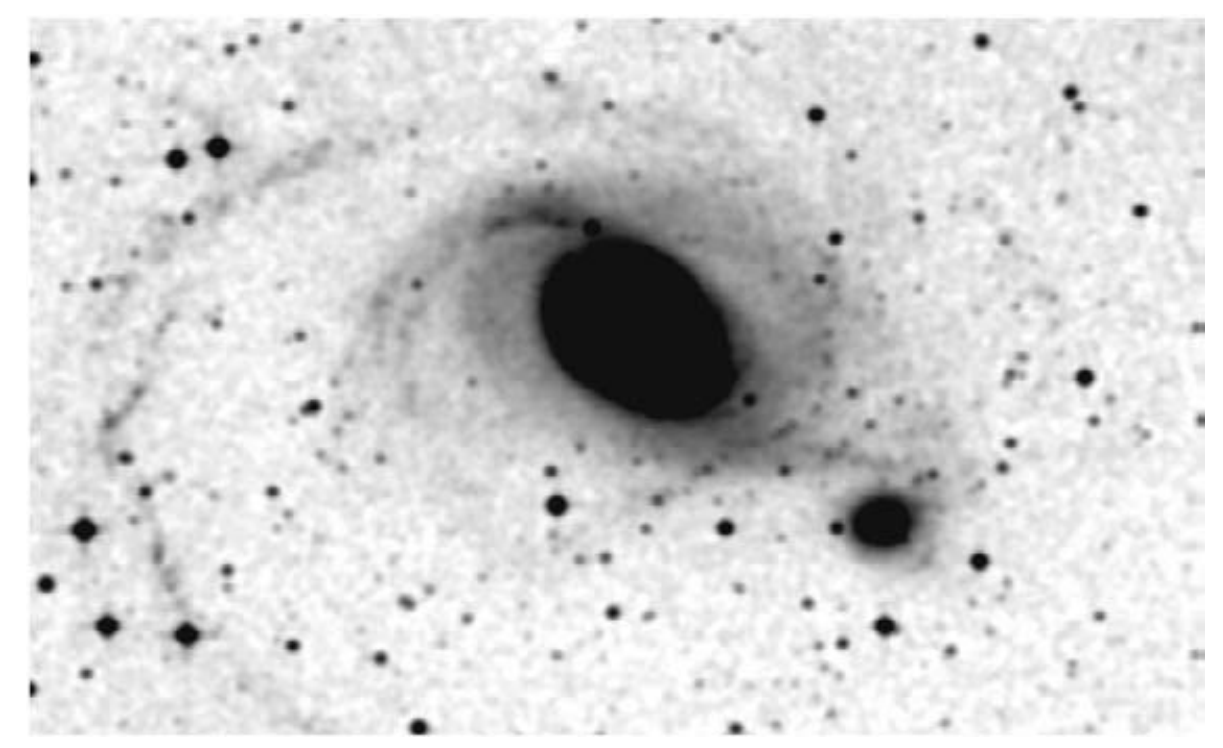

Figure 1. Digitized Sky Survey (DSS) image of the NGC 1512 spiral and NGC 1510 companion galaxy (below right) showing the luminous bridge connecting the two systems. Similar bridges are seen in HI and stars in other starburst satellites.

Ott et al. 2003) and averaging over multiple bursts (Gilmore \& Wyse 1991) can lead to solar or even subsolar $\alpha$-element to iron ratios, as observed in M32.

\section{Evolution of the System}

The long term evolution of the accreting dwarfs should be quite interesting. They initially will grow in mass, and their dynamical friction time scales $\left(t_{d f} \propto M_{\text {sat }}^{-1}\right)$ at first get shorter (e.g., Taylor \& Babul 2001). But eventually competing effects must come into play, which may include exhaustion of the outer disk gas supply for accretion and the beginnings of tidal mass loss as the orbital radius shrinks. At this point we expect that mass growth will cease, and the companion galaxy would enter a phase of its evolution primarily driven by dynamical processes: tidal friction and tidally induced mass loss (see Kravtsov et al. 2004).

Satellite galaxies orbiting with low inclinations to a disk in prograde directions are not only well positioned to capture gas, but also lose angular momentum relatively rapidly due to resonant coupling to the disk (Walker et al. 1996). As a result, their dynamical friction time scales are reduced. If the satellite moves inwards, then the kinds of tidal effects invoked in galaxy stripping models (Nieto \& Prugniel 1987) become increasingly important. However, in cases where earlier star formation produced high density central regions, the galaxy will be quite durable and a well-defined remnant can persist into the final phases of the capture process.

This extension of the transformed galaxy model differs from the purely tidal models in suggesting that under some circumstances companion galaxies can grow to high central densities that are fed by gas capture and subsequent starburst. Thus the system that enters into the final capture by its host is already unusually dense, a requirement for the M32 progenitor (Choi et al. 2002). This factor then joins the time scale arguments put forward by Nieto \& Prugniel to explain the rarity of M32-type compact dwarf ellipticals. Moderate mass galaxies will have orbital lifetimes of at most a few Gyr after orbiting 


\section{NGC1510: WFPC2 Central Starburst}

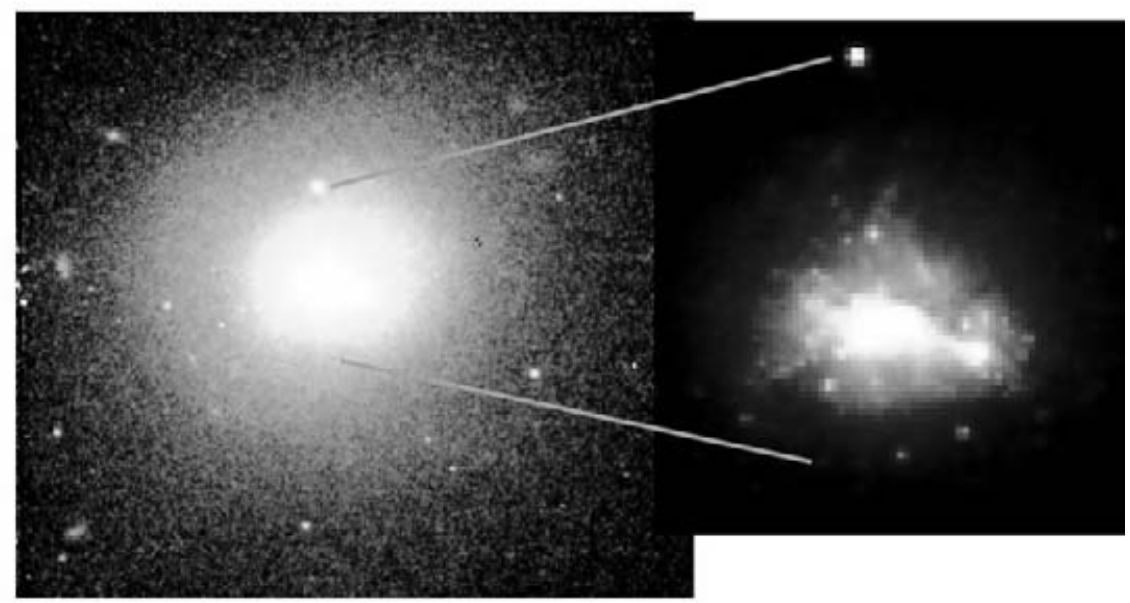

Figure 2. WFPC2 I-band image of the center of NGC 1510 showing the central concentration of star formation. The vertical space between the lines is 14 arcsec corresponding to $680 \mathrm{pc}$ for $\mathrm{D}=10 \mathrm{Mpc}$ to the system. This centrally condensed star forming pattern is also seen in other interacting companion galaxies.

close enough to their massive hosts to be heavily tidally stripped in their outer regions, thereby assuming compact morphologies.

\section{Discussion and Open Issues}

This M32 model resembles that of Bekki et al. (2001) in invoking special circumstances to drive gas inwards as fuel for a central starburst. The main difference is our assertion that gas capture is likely to play a role, which we believe is observationally supported. Indeed it seems reasonable that any model that produces concentrated starbursts in satellite galaxies sets the stage for the eventual formation of M32-like tidal remnants.

A second factor in favor of the capture model is the amount of gas that would be required to make the high density M32 galaxy. If we assume that the gas lost via winds about equals the amount of gas put into stars, then we need to have fed $\geqslant 3 \times 10^{9} \mathrm{M}_{\odot}$ of gas into the center of the progenitor system. This is a challenge for any model, though the outer gas reservoirs of major spirals, especially in their youth, may have been up to the task.

Naturally several issues remain to be resolved, including: (1) The central mass densities and stellar velocity dispersions in starburst satellite galaxies should be measured and compared with those in compact dwarf Es. We also need star formation rate determinations to assess time scales for stellar mass growth in their inner regions. (2) These are the galaxies with the highest baryonic densities. What sets this limit? And does the central black hole in M32 play a role in this? (3) The impact of extensive mass exchanges between satellite galaxies and the outer regions of their spiral galaxy hosts has yet to be considered. (4) What mass hosts are necessary to produce M32-type remnants under this model? For example, the Bekki et al. (2001) models required relatively massive spiral 
precursors, while M82 could be an example of a moderate mass precursor that has grown through gas capture. (5) Is the orbit of M32 suitable for gas capture, or nearly polar as adopted by Bekki et al. (2001)? (6) Additional numerical modeling of the circumstances under which dwarf galaxies can efficiently capture gas would be very helpful. In particular, these might allow us to understand the conditions, if any, under which late gas accretion could occur into the predicted large numbers of CDM mini-halos.

\section{Acknowledgements}

Thanks go to Bruno Binggeli and Helmut Jerjen for arranging this excellent workshop. We thank Tabetha Hole for helpful comments on this paper. JSG's research on dwarf galaxies is supported in part by NASA through grants administered by the Space Telescope Science Institute in conjunction with U.S. general observer programs, and by the National Science Foundation through grant AST-9803018 to the University of Wisconsin.

\section{References}

Bekki, K., Couch, W.J., Drinkwater, M.J. \& Gregg, M.D. 2001, ApJ (Letters) 557, L39

Burkert, A. 1994, MNRAS 266, 877

Choi, P.I., Guhathakurta, P. \& Johnston, K.V. 2002, AJ 124, 310

Duc, P.-A., Bournaud, F. \& Masset, F. 2004, A\&A 427, 803

Faber, S.M. 1973, ApJ 179, 423

Fellhauer, M. \& Kroupa, P. 2005, MNRAS 359, 223

Gilmore, G. \& Wyse, R.F.G. 1991, ApJ (Letters) 367, L55

Hawarden, T.G., van Woerden, H., Goss, W.M., Mebold, U. \& Peterson, B.A. 1979, A\&A 76, 230

Joseph, C.L. et al. 2001, ApJ 550, 668

Lauer, T.R., Faber, S.M., Ajhar, E.A., Grillmair, C.J. \& Scowen, P.A. 1998, AJ 116, 2263

Kravtsov, A.V., Gnedin, O.Y. \& Klypin, A.A. 2004, ApJ 609, 482

Miwa, T. \& Noguchi, M. 1998, ApJ 499, 149

Nieto, J.-L. \& Prugniel, P. 1987, A\& $A$ 186, 30

Ott, J., Martin, C. \& Walter, F. 2003, ApJ 594, 776

Rose, J.A., Arimoto, N., Caldwell, N., Schiavon, R.P., Vazdekis, A. \& Yamada, Y. 2005, AJ 129, 712

Taylor, J.E. \& Babul, A. 2001, ApJ, 559, 716

Walker, I.R., Mihos, C. \& Hernquist, L. 1996 ApJ 460, 121

Worthey, G. 2004, AJ 128, 2826

Worthey, G., Mateo, M., Alonso-García, J. \& España, A.L. 2004, PASP 116, 295

Young, L. 2000, $A J$ 119, 188

\section{Discussion}

LIN: With the shallow potential of satellite galaxies it is difficult to accrete gas unless the relative speed between them and the gas is less than the internal velocity dispersion.

Gallagher: Agreed. Only satellites on prograde orbits, presumable near the disk plane, should be able to accrete much gas. One caveat is how dwarfs interact with tidal spiral arms - this may increase accretion efficiencies.

MIESKE: Would you expect formation of compact dEs via gas accretion in very massive clusters, where you have high relative velocities?

Gallagher: No, because you need low relative velocities. Ram-pressure stripping seems to be the key factor for evolution of cdEs in massive clusters. 